\title{
Relação entre estresse e isolamento social durante a pandemia do Sars-Cov-2 em discentes de medicina
}

\author{
Stress related to the social isolation during the Sars-Cov-2 pandemic in students of a \\ medical school
}
Relación entre estrés y aislamiento social durante la pandemia Sars-Cov-2 en estudiantes de medicina

Ana Laura Lopes da Silva ${ }^{1}$, Daniela Silveira Marques Branco ${ }^{1 *}$, Ellen Pedroso Oliveira de Paula ${ }^{1}$, Júlia de Oliveira Sacchi ${ }^{1}$, Luana Carolina Rodrigues Guimarães ${ }^{2}$.

\section{RESUMO}

Objetivo: Avaliar o estresse e correlacionar com o isolamento social pela pandemia em discentes do Curso de Medicina em universidade do interior do estado de São Paulo. Métodos: Estudo observacional, transversal, descritivo e analítico. Aplicou-se o Inventário de Sintomas de Estresse para Adultos de Lipp (ISSL) de forma online, para identificação de sintomas de estresse na população de estudantes do Curso de Medicina do $1^{\circ}$ ao $6^{\circ}$ ano, associado ao questionário coletou-se informações relacionadas ao perfil sócio demográfico e status de isolamento de cada indivíduo. Estudo aprovado por Comitê de Ética em Pesquisa. Resultados: Amostra constituída por 170 discentes, sendo $77,1 \%$ do sexo feminino e $22,9 \%$ do sexo masculino, com maior proporção de alunos entre 18 e 25 anos. $72,9 \%$ consideraram estarem parcialmente em isolamento social, $19,4 \%$ em isolamento total e 7,6\%\% não estavam em isolamento social. Dos 170 participantes $67,6 \%$ apresentaram algum sintoma de estresse, enquanto $32,4 \%$ não manifestaram sintomas suficientes para classificação em alguma fase de estresse. Conclusão: Conclui-se que alunos do curso tem elevados níveis de estresses, explicado por inúmeros fatores, mas no decorrer do ano de 2020 apresentaram índices ainda mais altos, tendo relação paralela com diminuição do contato social e sobrecarga das atividades online.

Palavras-chave: Pandemia, Estresse, Isolamento social.

\begin{abstract}
Objective: To assess stress and correlate it with social isolation caused by the pandemic in medical students at a university in the interior of the state of São Paulo. Methods: Observational, cross-sectional, descriptive and analytical study. Lipp's Inventory of Stress Symptoms for Adults (ISSL) was applied online to identify stress symptoms in the population of medical students from 1st to 6th year, associated with the questionnaire collected related information the socio-demographic profile and isolation status of each individual. Study approved by the Research Ethics Committee. Results: Sample made up of 170 students, $77.1 \%$ female and $22.9 \%$ male, with a higher proportion of students between 18 and 25 years old. $72.9 \%$ considered they were partially in social isolation, $19.4 \%$ in total isolation and $7.6 \%$ were not in social isolation. Of the 170 participants, $67.6 \%$ had some symptom of stress, while $32.4 \%$ did not show enough symptoms to classify them in some phase of stress. Conclusion: But over the course of 2020 they had even higher rates, having a parallel relationship with a decrease in social contact and overload of online activities.
\end{abstract}

Keywords: Pandemic, Stress, Social isolation.

\section{RESUMEN}

Objetivo: Evaluar el estrés y correlacionarlo con el aislamiento social causado por la pandemia en estudiantes de medicina de una universidad del interior del estado de São Paulo. Métodos: Estudio observacional, transversal, descriptivo y analítico. Se aplicó en línea el Inventario de Síntomas de Estrés en Adultos (ISSL) de Lipp para identificar los síntomas de estrés en la población de estudiantes de medicina de $1^{\circ}$ a $6^{\circ}$ año, asociado al cuestionario recogido información relacionada con el perfil sociodemográfico y el estado de

1 Universidade de Franca (UNIFRAN), Franca - SP. *E-mail: daniela_silveira2003@hotmail.com

2 Hospital Escola de Itajuba (AISI-FMIT), Itajuba - MG.

SUBMETIDO EM: 8/2021 
aislamiento de cada individuo. Estudio aprobado por el Comité de Ética en Investigación. Resultados: Muestra compuesta por 170 estudiantes, $77,1 \%$ mujeres y 22,9\% hombres, con una mayor proporción de estudiantes entre 18 y 25 años. El 72,9\% consideró que estaban parcialmente en aislamiento social, el 19,4\% en total aislamiento y el 7,6\% no estaban en aislamiento social. De los 170 participantes, el 67,6\% presentaba algún síntoma de estrés, mientras que el 32,4\% no mostraba síntomas suficientes para clasificarlos en alguna fase de estrés. Conclusion: Pero a lo largo de 2020 tuvieron índices aún mayores, teniendo una relación paralela con la disminución del contacto social. y sobrecarga de actividades en línea.

Palabras clave: Pandemia, Estrés, Aislamiento social.

\section{INTRODUÇÃO}

Coronavírus é um vírus zoonótico, da família Coronaviridae, responsáveis por infecções respiratórias. Ao final de 2019 descreveu-se o novo coronavírus na cidade de Wuhan, na China, provocando a doença COVID19, a qual atingiu enormes proporções, sendo que em 11 de março de 2020 a Organização Mundial da Saúde (OMS) declarou pandemia. Notificou-se o caso um no Brasil em 26 de fevereiro de 2020 e no dia 17 de março o primeiro óbito (LIMA CMO, 2020). Nesse mesmo mês alguns estados começaram a estipular as medidas de quarentena, consistindo no fechamento de estabelecimentos não essenciais e recluindo as pessoas em casa, evitando o contato social (OLIVEIRA WK, et al., 2020).

Diante de tal situação, a Secretaria da Educação do Estado de São Paulo decretou à suspensão das aulas presenciais, assim, as escolas e universidades deveriam se adaptar para desenvolver as atividades de forma online (SECRETÁRIA DE EDUCAÇÃO, 2020). Entretanto, isso trouxe uma intensa mudança na rotina desses alunos, ao ficarem reféns da tecnologia e sujeitos a alterações dos padrões do ensino, logo mais propensos ao desenvolvimento de sinais e sintomas de estresse, e, por conseguinte uma diminuição da qualidade de vida, por se sentirem "prisioneiros" em sua própria residência e sobrecarregados com as diferentes tarefas (SCHMIDT B, et al., 2020).

Pode-se caracterizar o estresse como um estado de percepção de estímulos que provocam excitação emocional, desencadeados por um estressor (MARGIS R, et al. 2003). Sua evolução consiste em três fases, sendo a de alerta (contato com o agente estressor), resistência (corpo busca o equilíbrio) e exaustão (comprometimento físico em forma de doença) (MINISTÉRIO DA SAÚDE, 2015; ZHU N, et al., 2020). Além disso, pode-se ainda determinar o estresse em indicadores, sendo eles físico, emocional, comportamental e/ou cognitivo (ENUMO SRF, et al., 2020).

Da mesma forma cabe ressaltar o conceito de saúde mental, que segundo a Organização Mundial da Saúde (OMS) é um estado de bem-estar em que o indivíduo consegue-se adaptar ao estresse cotidiano esperado, sendo capaz de exercer funções diárias e trabalhistas (WHO, 2018). Contudo com a intensidade das infecções pelo vírus associado a todo estresse e pressão que as pessoas são submetidas, fica claro que tal conceito torna-se cada vez mais preocupante (PRADO AD, et al., 2020)

Assim, as condições atuais afetam o bem-estar psicológico das pessoas ao identificar sintomas de ansiedade e estresse diante do expressivo contágio do vírus, seja pelo próprio medo de contrair a doença ou então de contaminação dos entes queridos; assim como, as preocupações com escassez de suprimento alimentar, devido às perdas financeiras (SCHMIDT B, et al., 2020). Ademais, o fechamento de escola e universidades acarreta a diminuição de conexões face a face, de interações sociais e acúmulo de tarefas "homeschooling" contribuindo como forte fator estressor, o que exacerba as emoções negativas, como tristeza, medo, solidão e raiva (AFIFI WA, et al., 2012).

Associado a isso, cabe ressaltar o papel da mídia, que diferentemente de acontecimentos do passado, as pessoas não tinham muito conhecimento da intensidade das pandemias, logo muitas mensagens verdadeiras ou não, "fake news", contribuem para o pânico geral (SCHMIDT B, et al., 2020). Dessa forma, o impacto psicológico tende a ser inevitável em muitos casos, não apenas aos estudantes, mas para toda a população de forma geral, com ênfase em cientistas e profissionais de saúde que são cobrados pela busca de respostas a uma terapêutica eficaz ou por uma cura definitiva (LIMA SO, et al., 2020). Em meio a isso, já foram até reportados casos de suicídio na Coreia do Sul e Índia, demonstrando a gravidade da situação (SCHMIDT B, et al., 2020). 
Devido a inconstância desse período pandêmico tais consequências mentais podem ser explicadas pela Teoria do Sistema Imune Comportamental (BIS), em que pensamentos negativos aparecem como um "escudo protetor", acarretando em comportamentos obsessivos por normas sociais especificas. A princípio tais atitudes podem afastar o indivíduo do vírus, entretanto, em um futuro não distante pode prejudicar o sistema imunológico da pessoa, tornando a propensa a enfermidades mentais, como o Transtorno do Pânico, Transtorno de Estresse Pós-Traumático (TEPT) e Transtorno Obsessivo Compulsivo (TOC) (LIMA SO, et al., 2020).

Cabe ressaltar que pacientes com esquizofrenia apresentaram alta mortalidade e maiores taxas para desenvolvimento de outras enfermidades, além de manifestarem quadro clínico mais grave ao SARS-COV-2. Assim como a população idosa, em que as condições psiquiátricas foram acentuadas, com ênfase em mulheres que possuem predisposição maior a depressão, ansiedade, estresse e TEPT (LIMA SO, et al., 2020).

Em linhas gerais a orientação de isolamento social trouxe aumento no número de denúncias de casos de violência doméstica no Brasil. O enclausuramento associado a redução da fonte financeira e da alimentação, aumento do uso de álcool e drogas ilícitas induziu a agressividade, tornando os grupos sociais vulneráveis, como a mulher, adolescentes e crianças (BAGGENSTOSS GA, et al., 2020).

Intervenções possíveis para amenizar essa situação encontram-se nos cuidados com o sono, busca por práticas de atividades físicas e técnicas de relaxamento, além das propostas psicoeducativas, como cartilhas, canais de escuta psicológica ou então atendimentos em plataformas online (SCHMIDT B, et al., 2020). A "Cartilha para enfrentamento do estresse em tempos de pandemia" de autoria da OMS tem como objetivo promover conhecimento científico de forma mais coloquial, de forma que facilite a acessibilidade de todos; além disso, mostra que saúde mental não é somente a ausência de transtornos mentais, mas também capacidade de adaptação ao estresse e o equilíbrio com o bem-estar emocional (ENUMO SRF, et al., 2020; KEYES CL, 2007).

Outras formas de amenizar esses julgamentos danosos são por meio de cultos religiosos online, confortando os fiéis, assim como, as mídias sociais devem emitir notícias positivas referentes ao enfrentamento da pandemia e os profissionais de saúde (psicólogos e psiquiátricas) devem realizar lives para auxiliar a população. Portanto, tais intervenções são essenciais pois danos no estado mental são altamente prevalentes e apresentam grandes alterações futuras (LIMA SO, et al., 2020).

O estudo teve como objetivo avaliar o estresse e correlacionar com o isolamento social pela pandemia por Sars-cov-2 de discentes do curso de medicina de uma universidade no interior de São Paulo no ano de 2020. Além de verificar o estresse pelo instrumento Inventário de Sintomas de Stress para Adultos de Lipp (ISSL), durante a pandemia do COVID-19 e descrever as fases e os sintomas do estresse, comparando-os com o perfil sociodemográfico e 0 ano de graduação do estudante.

\section{MÉTODOS}

\section{Delineamento de pesquisa e aspectos éticos}

Estudo observacional, transversal, descritivo e analítico das fases e dos sintomas do estresse em discentes do curso de medicina. Frente ao isolamento social elaborou-se um link com o questionário denominado, Inventário de Sintomas de Estresse para Adultos de LIPP (ISSL), que foi enviado aos alunos do curso, junto com o Termo de Consentimento Livre e Esclarecido (TCLE), além disso obteve-se autorização da Instituição de ensino. Todos os termos e projeto foi submetido ao Conselho de Ética e Pesquisa, obtendo aprovação (CAAE: 35760620.3.1001.5495).

O questionário aplicado contém três quadros, o primeiro quadro composto por 15 itens, destes 12 sintomas físicos e 3 psicológicos, referentes aos sintomas que o entrevistado tenha experimentado nas últimas vinte e quatro horas, o segundo quadro composto por 10 sintomas físicos e 5 psicológicos experimentados na última semana e no terceiro quadro composto por 12 sintomas físicos e 11 psicológicos referentes aos sintomas do último mês. No final, totaliza 37 itens de natureza somática e 19 itens de natureza psicológica. Cabe ressaltar que, desde o início contou-se com a supervisão de uma psicóloga, a qual também analisou os resultados. 


\section{Coleta de dados}

Analisaram-se 234 respostas de alunos do curso de Medicina de uma Universidade no interior do estado de São Paulo, das quais 64 pessoas foram excluídas por fazerem acompanhamento com psiquiatra, sendo um dos critérios de exclusão do estudo, resultando em 170 pessoas.

\section{Tratamento dos dados e análise estatística}

A análise estatística deste estudo foi descritiva e inferencial. As variáveis independentes foram aquelas relacionadas ao perfil sociodemográfico (sexo, faixa etária e estado civil) e ao ano da graduação no curso de medicina (primeiro ao sexto anos) e ao isolamento social devido a pandemia pelo vírus Sars-Cov-2 (não estavam em isolamento, estavam parcialmente em isolamento e estavam totalmente em isolamento). As variáveis dependentes foram as fases do estresse pela classificação de Lipps (alerta, resistência e exaustão) e aos sintomas do estresse (físicos, psicológicos ou ambos).

As variáveis qualitativas foram descritas por suas frequências absolutas e relativas. Em seguida a estatística inferencial foi efetuada por meio do teste Qui-quadrado $\left(\chi^{2}\right)$ que foi de 33,845 e valor $p<0,001$ para comparação de frequências.

Os dados foram planificados no editor de planilhas Excel da Microsoft. Para todas as análises estatísticas foram considerados o nível de significância de $5 \%$ (com grau de significação de $p<0,05$ ) e foram realizadas pelo auxílio do programa computacional Statistical Packageof Social Science (SPSS) versão 26.0.0.

\section{RESULTADOS}

\section{Descrição da amostra}

A amostra foi constituída por 170 discentes do primeiro ao sexto ano do curso de Medicina da Universidade, sendo 131 (77,1\%) do sexo feminino e 39 (22,9\%) do sexo masculino. Em relação à faixa etária, $2(1,2 \%)$ é menor de 18 anos, $147(86,2 \%)$ possuem entre 18 e 25 anos, $18(10,6 \%)$ entre 26 e 30 anos e 3 $(2,4 \%)$ possui mais de 30 anos. No quesito estado civil, $4(2,4 \%)$ pessoas se encontravam casadas ou em união estável e 166 estavam solteiras, sendo destas 80 (47,1\%) possuíam vínculo amoroso e 86 (50,6\%) não possuíam vínculo amoroso. A respeito do ano de graduação, 22 (12,9\%) alunos estavam no primeiro ano, 27 no segundo ano, 21 no terceiro ano, 58 no quarto ano, 19 no quinto ano e 23 no sexto ano (Tabela 1).

Tabela 1 - Distribuição das frequências dos discentes de acordo com o sexo, com a faixa etária, com o estado civil e com o ano de graduação no curso de Medicina ( $n=170)$.

\begin{tabular}{llcc}
\hline Variável & Descrição da variável & $\begin{array}{c}\text { Frequências absolutas } \\
\text { (n) }\end{array}$ & $\begin{array}{c}\text { Frequências relativas } \\
(\%)\end{array}$ \\
\hline \multirow{2}{*}{ Sexo } & Feminino & 131 & 77,1 \\
& Masculino & 39 & 22,9 \\
\hline \multirow{4}{*}{ Faixa etária } & Menor que 18 anos & 2 & 1,2 \\
& Entre 18 e 25 anos & 147 & 86,2 \\
& Entre 26 e 30 anos & 18 & 10,6 \\
& Maior que 30 anos & 3 & 1,8 \\
\hline \multirow{3}{*}{ Estado civil } & Casado ou em união estável & 4 & 2,4 \\
& Solteiro e com vínculo amoroso & 80 & 47,1 \\
& Solteiro e sem vínculo amoroso & 86 & 50,6 \\
\hline \multirow{5}{*}{ Ano de graduação } & Terceiro & 22 & 12,9 \\
& Quarto & 27 & 11,9 \\
& Quimeiro & 21 & 12,4 \\
& Segundo & 58 & 34,1 \\
& Sexto & 19 & 11,2 \\
\hline
\end{tabular}

Fonte: Silva ALL, et al., 2021. 
A pesquisa foi realizada entre os dias 26 de agosto e 2 de setembro de 2020, período em que os alunos do primeiro ao quarto ano se encontravam em um ensino totalmente à distância e os alunos do quinto e sexto ano já haviam retornado à maioria de suas atividades práticas. Levando isso em conta, foi encontrado uma taxa de $7,6 \%$ de alunos que não estavam em isolamento social, $72,9 \%$ que se encontravam em isolamento social parcial e 19,4\% em isolamento social total (Figura 1).

Figura 1 - Distribuição das frequências dos discentes do curso de Medicina em isolamento social devido à pandemia pelo vírus Sars-cov-2 no ano de 2020.

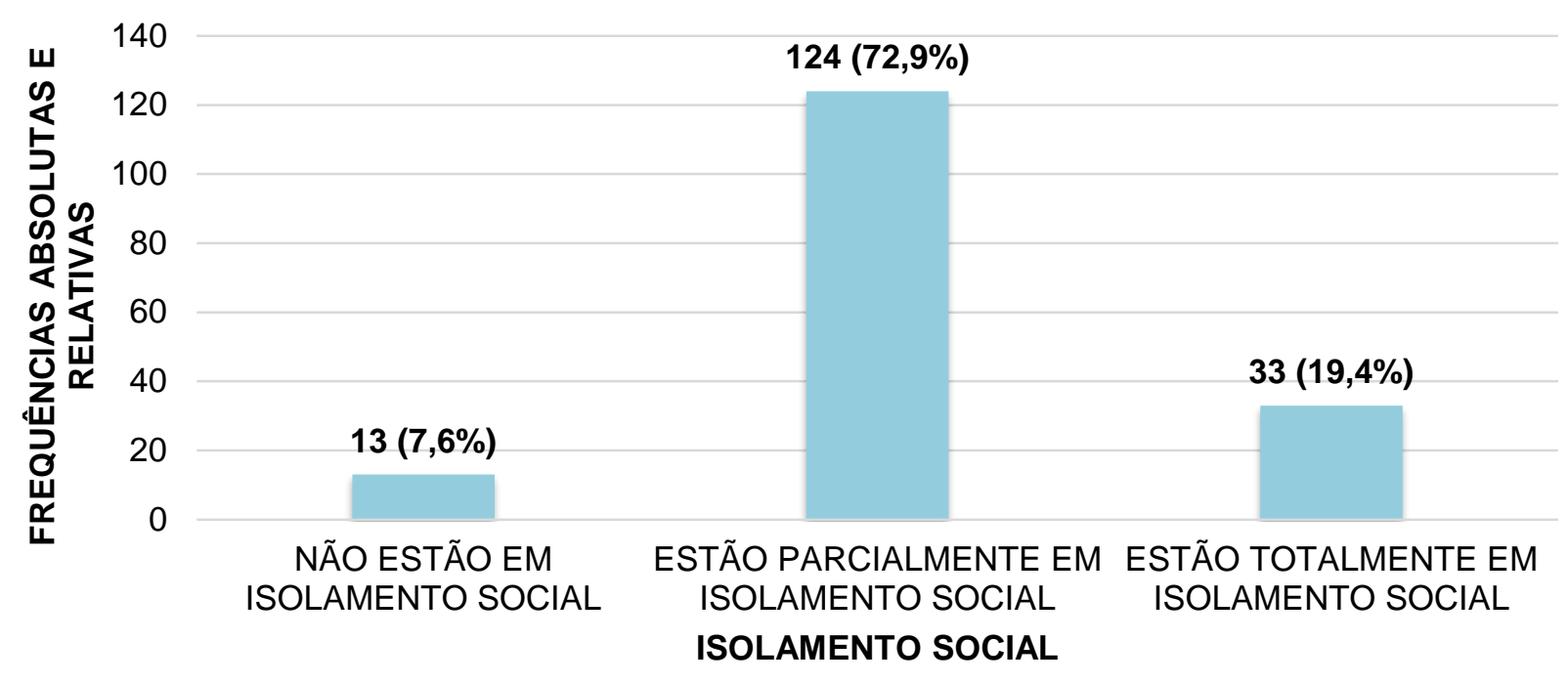

Fonte: Silva ALL, et al., 2021.

\section{Estudantes com e sem estresse e prevalência de sinais e sintomas psicológicos ou físicos}

Dos 170 alunos participantes, 99 (58,2\%) apresentam sintomas psicológicos de estresse e 112 (65,9\%) apresentavam sintomas físicos de estresse, através do teste ISSL (Figura 2).

Figura 2 - Distribuição das frequências dos discentes do curso de Medicina que apresentaram sintomas psicológicos e físicos do estresse no ano de $2020(n=170)$.

\section{SINTOMAS PSICOLÓGICOS}

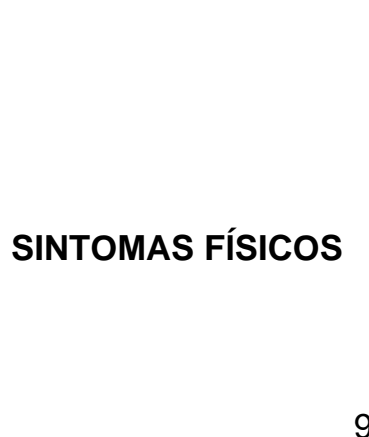

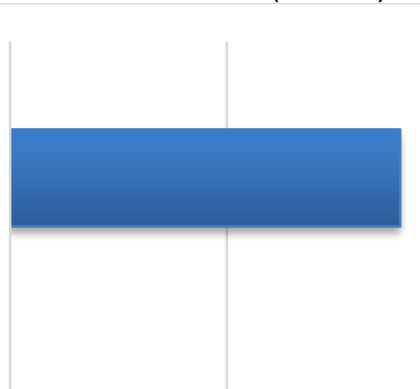

$99(58,2 \%)$
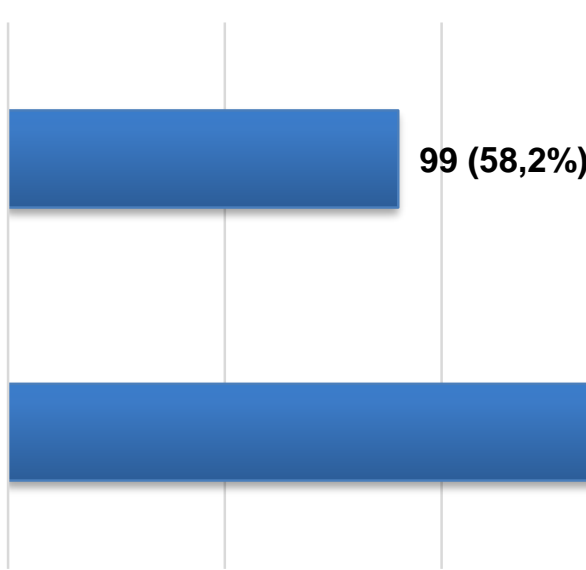

90
$112(65,9 \%)$

\section{5 \\ FREQUÊNCIAS ABSOLUTAS E RELATIVAS}

Dos 170 participantes desta pesquisa, 67,6\% $(n=115)$ apresentaram algum sintoma de estresse e 32,4\% $(n=55)$ não manifestaram sinais e sintomas suficientes para serem classificados nas fases do estresse. 
Dessa forma, dentre os 115 alunos, $10 \%(n=17)$ encontravam-se na fase de alerta (contato com o agente estressor), 39,4\% ( $n=67)$ na fase de resistência (corpo busca o equilíbrio) e 18,2\% $(n=31)$ na fase de exaustão (comprometimento físico em forma de doença) (Figura 3).

Figura 3 - Distribuição das frequências dos discentes do curso de Medicina em fases de alerta, de exaustão e de resistência do estresse no ano de 2020.

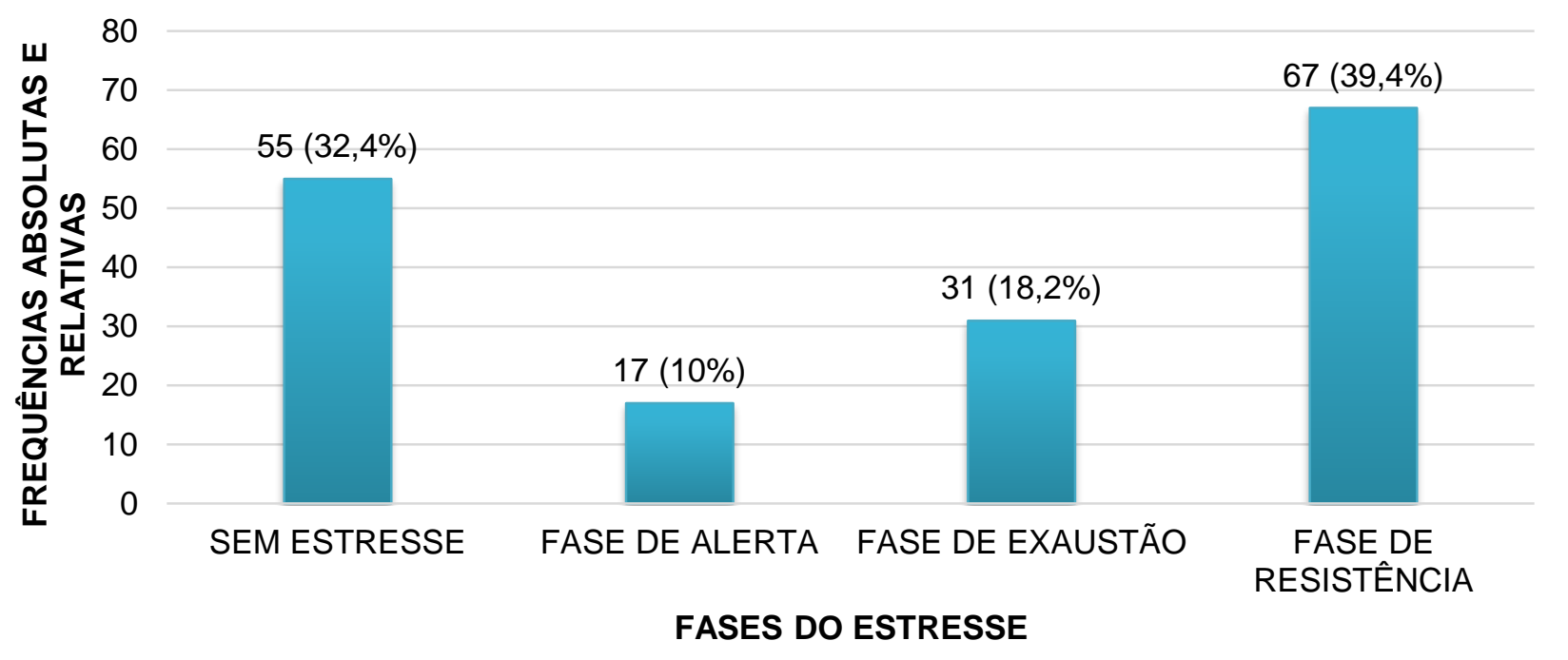

Fonte: Silva ALL, et al., 2021.

Ao analisar o nível de estresse dos alunos com base em seus respectivos anos de graduação, apesar da discrepância em número entre as amostras, observa-se que o estresse tende a diminuir com o passar dos anos, ou seja, no primeiro ano apenas $28 \%$ dos alunos não tinham nenhum tipo de estresse e no sexto ano $55 \%$ dos alunos; logo, é possível encontrar uma relação estatística entre esses dois parâmetros, nível de estresse e ano de graduação, com valores de qui-quadrado de 33,845 e p < 0,001 (Figura 4).

Figura 4 - Distribuição das frequências absolutas e relativas das fases de estresse de acordo com o ano de graduação dos alunos do curso de Medicina.

\section{FREQUÊNCIAS ABSOLUTAS E RELATIVAS DAS FASES DO ESTRESSE DE ACORDO COM O ANO DE GRADUAÇÃO}

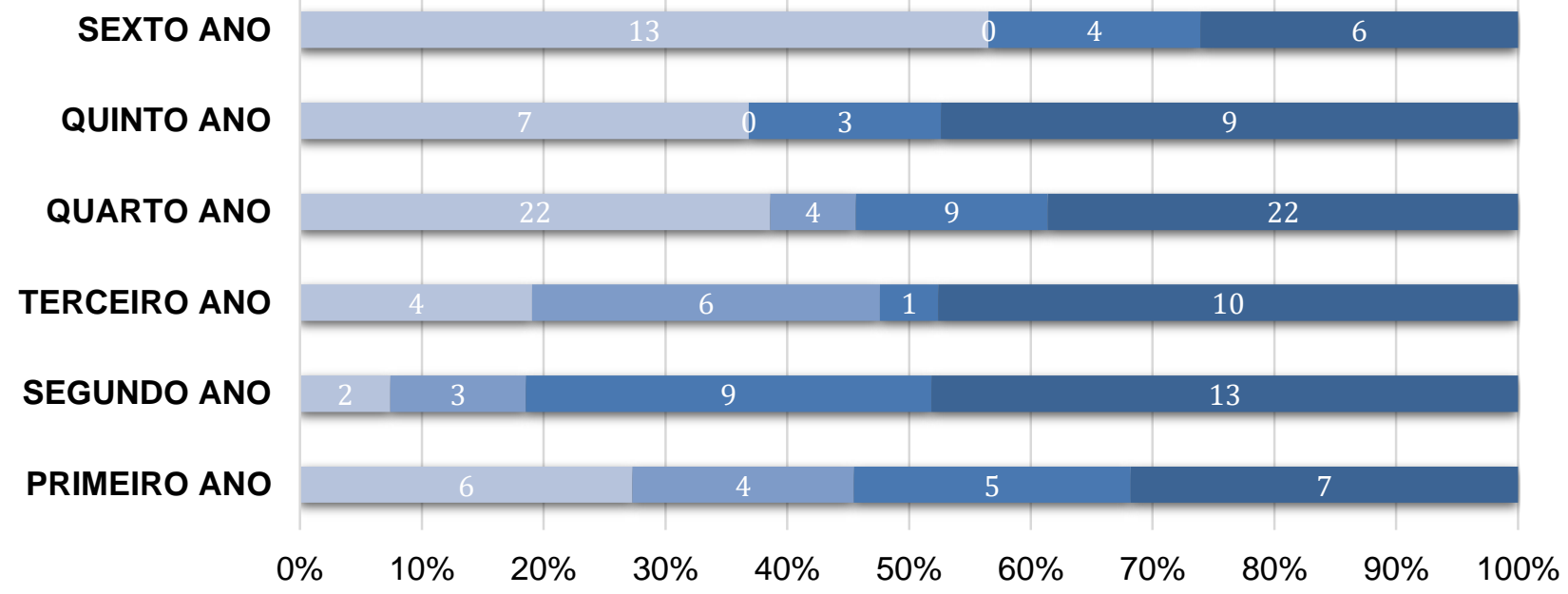

SEM ESTRESSE FASE DE ALERTA —FASE DE EXAUSTÃO \#FASE DE RESISTÊNCIA

Fonte: Silva ALL, et al., 2021. 


\section{DISCUSSÃO}

Estudo realizado com amostra de 170 participantes do $1^{\circ}$ ao $6^{\circ}$ ano de uma Universidade de Medicina no interior de São Paulo, com predomínio no sexo feminino, frequência relativa de $77 \%$, e $23 \%$ das respostas do sexo masculino. Segundo dados no Instituto Nacional de Estudos e Pesquisas Educacionais Anísio Teixeira (INEP) 2018 o curso de medicina está entre os vinte maiores cursos em número de matrículas, sendo que 87.122 alunos correspondem ao sexo feminino e 62.523 do sexo masculino (MINISTÉRIO DA EDUCAÇÃO, 2017).

A variação de faixa etária que houve maior predomínio encontra-se entre 18 e 25 anos, correspondendo a 147 candidatos, com uma porcentagem de $86 \%$; seguida de participantes com idade entre 26 e 30 anos, correspondendo a 18 candidatos, com uma porcentagem de 10\%; 3 alunos maiores de 30 anos, representando 1,8\%; e apenas 2 alunos com menos de 18 alunos, representando 1,2\%. Essa faixa etária também foi evidenciada como a mais prevalente entre os estudantes de medicina na Universidade do Estado do Rio Grande do Norte, que demonstrou 76,4\% dos alunos entre a faixa de 18 a 24 anos (CARDOSO FILHO FAB, et al., 2015; REZENDE CHA, et al., 2007).

Ao analisar a frequência de estresse em relação ao ano de ensino é possível inferir que os alunos que ainda estão cursando a primeira metade do curso apresentam maior nível de estresse que os alunos que já estão na segunda metade. Esse fato é evidenciado por $28 \%$ dos alunos do primeiro ano não apresentarem estresse em comparação à $55 \%$ dos alunos do sexto ano não apresentarem estresse $(p<0,001)$. Fica claro que exigências se iniciam antes do ingresso no curso, sendo a graduação em Medicina um dos cursos mais concorridos do exame vestibular, logo acadêmicos de Medicina apresentam níveis de estresse mais elevados do que a população geral e esse pode aumentar principalmente ao longo do primeiro ano universitário (LIMA RL, et al., 2016).

Acredita-se que níveis de estresse mais elevados no primeiro tenha ampla relação com questões adaptativas, e além disso, os dois primeiros anos de graduação são compostos pelo "ciclo básico", com predomínio de atividades teórico-práticas e menor parcela de conteúdos curriculares clínicos. O terceiro ano apresenta intersecção entre o ciclo básico e o clínico, em que começa a notar melhora do estresse entre os alunos (VASCONCELOS TC, et al., 2015).

Associado ao exposto alguns autores sugerem que os principais problemas enfrentados no ciclo básico são a dificuldade em se adequar a nova rotina, solidão, distânciamento dos familiares, ausência de atividades de lazer, início de novas amizades e disputa pela obtenção de melhores notas; por outro lado na segunda metade do curso os fatores estressores constituem a angustia de possível fracasso, preocupação pelo aumento de atribuições e responsabilidades, ansiedade e insegurança ao realizar exames clínicos, temor de contaminação por doenças infectocontagiosas e afastamento dos colegas (MOREIRA SNT, et al., 2015).

As fases do estresse, pela Classificação de Lipp, mais prevalente foi a fase de resistência $(39,4 \%)$, seguida da fase de exaustão $(18,2 \%)$ e por fim a fase de alerta (10\%). Em relação aos sintomas, os mais frequentes foram os físicos, presentes em $65,9 \%$ dos participantes.

Estudo realizado no ano de 2016 com amostra de 456 alunos do $1^{\circ}$ ao $4^{\circ}$ ano do curso de medicina da Universidade Federal do Paraná ao aplicar o Inventário de Sintomas de Estresse para Adultos de Lipp (ISSL) obteve-se como resultado ausência de estresse em apenas 182 (39,91\%) participantes, enquanto 274 $(60,09 \%)$ apresentavam algum tipo de estresse, sendo encontrado elevada taxa de alunos na fase de resistência (LIMA RL, et al., 2016). Outro estudo realizado na Faculdade de Medicina da Universidade Federal do Ceará no ano de 2009, que utilizou a mesma metodologia de uma amostra de 199 alunos, cerca de 99 $(49,7 \%)$ apresentaram algum tipo de estresse, sendo que $85,8 \%$ desses encontravam-se na fase de resistência (AGUIAR SM, et al., 2009; ZONTA R, et al., 2006).

Assim como no atual estudo, a fase de resistência apresentou índices mais elevados, entretanto, diferente dos alunos da universidade do interior de São Paulo que com o decorrer do curso o índice de estresse tende a diminuir, na Universidade Federal do Paraná, os valores tendem a aumentar (LIMA RL, et al., 2016). 
Contudo, diante do exposto fica claro que estudantes de medicina passam por um intenso processo de estresse durante os anos de faculdade, sendo que inúmeros fatores, pessoais e institucionais podem ser responsáveis. Dentre os fatores institucionais, como já mencionado, estão os diferentes ciclos do ensino médico. Dentre os fatores pessoais estão a adequação do aluno ao método de ensino e à instituição, rede de apoio construída durante os anos de convivência entre os alunos e a maior maturidade para enfrentar as alterações da metodologia durante o isolamento social (GONÇALVES MB e PEREIRA AMTB, 2009).

Em concordância ao analisar um estudo também realizado em uma universidade no interior de São Paulo no ano de 2018 com amostra de 166 alunos, sendo 83 do primeiro ano de graduação e 83 do segundo ano, ao aplicar o ISSL detectaram a presença de certo nível de estresse, comum nos cursos de medicina. Além disso, observou-se que o estresse diminuiu entre esses dois anos de graduação, encontrado porcentagem de $87,95 \%$ no primeiro ano e $75,90 \%$ no segundo $(p<0,05)$.

Assim, ao comparar o estudo de 2018 com o de 2020, utilizando os parâmetros do primeiro e segundo ano de graduação o nível de estresse mostrou-se maior no ano de 2020, com uma porcentagem relativa de $10 \%$ de diferença, o que pode ser justificado pela necessidade de isolamento social, o que estreitou as relações sociais, acompanhado do ensino a distância, que pode ser totalmente estressante aos alunos, ultrapassando então os limites de estresse esperado para um aluno de medicina.

Portanto, estudantes de medicina que se encontram estressados podem ter seus aprendizados afetados e como consequência, influir no cuidado com o paciente (LIMA RL, et al., 2016). Níveis elevados de estresse atrapalha no aprendizado, uma vez que interfere com funções executivas cerebrais, afetando o ensino em algum momento especifico, e se prolongado, pode afetar o desempenho durante todo o curso (FATEH R, et al., 2020). Com isso, os níveis de estresse dos alunos de medicina possuem consequências que vão além do âmbito pessoal, podendo prejudicar a educação do discente, e quando isso ocorre em grande escala, como é o caso da pandemia da COVID-19, passa a representar um problema de saúde pública por si só.

\section{CONCLUSÃO}

Diante dos fatos fica claro que o presente artigo encontrou níveis prejudiciais de estresse na grande maioria dos estudantes de medicina durante a pandemia do COVID-19 quando comparado à estudos realizados na mesma instituição dois anos antes. Assim, a pandemia apresentou inúmeros obstáculos sobre a qualidade de ensino e o aproveitamento dos alunos, sendo um deles o elevado nível de estresse dos discentes. Através desse estudo, foi possível inferir que apesar de todas as alterações por parte das instituições de ensino para tentar se adequar da melhor maneira, os alunos saíram lesados, seja em relação a saúde mental e física ou em relação ao aprendizado.

\section{AGRADECIMENTOS}

Os autores agradecem a universidade por todo o apoio fornecido e principalmente a todos os alunos que se prontificaram a responder o questionário aplicada na pesquisa.

\section{REFERÊNCIAS}

1. AFIFI W, et al. The impact of uncertainty and communal coping on mental health following natural disasters. Anxiety, Stress \& Coping, 2012; 37: e200065.

2. AGUIAR S, et al. Prevalência de Sintomas de Estresse nos Estudantes de Medicina. Jornal Brasileiro de Psiquiatria, Fortaleza-Ce, 2009, p.34-38.

3. BAGGENSTOSS GA, et at. Violência contra mulheres e a pandemia do COVID-19: Insuficiencia de dados oficiais e de respostas do estado brasileiro. Revista Direito Público, Brasília, 2020; 94(17): 336-363.

4. CARDOSO FILHO FAB, et al. Perfil do Estudante de Medicina da Universidade do Estado do Rio Grande do Norte. Revista Brasileira Educação Médica, Rio de Janeiro, 2015; 39(1): 32-40.

5. ENUMO S, et al. Enfrentando o estresse em tempos de pandemia: proposição de uma cartilha. Estudo de Psicologia, Campinas, 2020; 1-10.

6. FATEH R, et al. High Levels of Stress Causing Anxiety Amongst Medical Studentes. International Journal of integrative Medical Sciences, 2020; 867-874 
7. GONÇALVES MB, PEREIRA AN. Considerações sobre o Ensino Médico no Brasil: consequências afetivo-emocionais nos estudantes. Revista Brasileira Educação Médica, Maringa, 2009; 493-504.

8. KEYES CLM. Promoting and protecting mental health as flourishing: a complementary strategy for improving national mental health. American Psychologist, 2007; 95-108.

9. LIMA CMO. Informações sobre o novo coronavírus (COVID 19). Radiologia Brasileira: Colegiado Brasileiro de Radiologia e Diagnóstico por Imagem, Rio de Janeiro, 2020; 5-6.

10. LIMA R, et al. Estresse do Estudante de Medicina e Rendimento Acadêmico. Revista Brasileira Educação Médica, Curitiba, 2016; 678-684.

11. LIMA SO, et al. Impactos no comportamento e na saúde mental de grupos vulneráveis em época de enfrentamento da infecção COVID-19: revisão narrativa. Revista Eletrônica Acervo Saúde, 2020; Esp. 46: e4006.

12. MARGIS R, et al. Relação entre estressores, estresse e ansiedade. Revista de Psiquiatria, Rio Grande do Sul, 2003; $1-10$.

13. MINISTÉRIO DA EDUCAÇÃO. Secretária Estadual da Educação. Censo da Educação Superior: divulgação dos principais resultados. Divulgação dos principais resultados, Brasil, 2017. Disponível em: http://portal.mec.gov.br/index.php?option=com_docman\&view=download\&alias=97041-apresentac-a-o-censosuperior-u-Itimo\&ltemid=30192. Acesso em: 10 jan. 2021.

14. MINISTÉRIO DA SAÚDE. Estresse. São Paulo: Biblioteca Virtual em Saúde, Brasil, 2015. Disponível em: https://bvsms.saude.gov.br/dicas-em-saude/2068-estresse. Acesso em: 10 abr. 2020.

15. OLIVEIRA W, et al. Como o Brasil pode deter a COVID-19. Epidemiologia e Serviçoes de Saúde, Brasília, 2020, p.18.

16. MOREIRA SNT, et al. Estresse na Formação Médica: como Lidar com Essa Realidade. Revista Brasileira de Educação Médica, Rio Grande do Norte, 2015; 39(4): 558-564.

17. PRADO A, et al. A saúde mental dos profissionais de saúde frente à pandemia do COVID-19: uma revisão integrativa. Revista Eletrônica Acervo Saúde, 2020; Esp.46: e4128.

18. REZENDE C, et al. Prevalência de sintomas depressivos entre estudantes de medicina da Universidade Federal de Uberlândia. Revista Brasileira de Educação Médica, 2007; 315-323.

19. SECRETÁRIA DE EDUCAÇÃO. Documento Orientador - Atividades Escolares Não Presenciais. São Paulo: 2020. Disponível em: http://www.escoladeformacao.sp.gov.br/portais/Portals/84/docs/pdf/documento-orientador-atividadesescolares-nao-presenciais.pdf. Acesso em: 10 jun. 2020.

20. SCHMIDT B, et al. Saúde mental e intervenções psicológicas diante da pandemia do novo coronavírus (COVID 19). Estudos de Psicologia, 2020; 1-13.

21. VASCONCELOS T, et al. Prevalência de Sintomas de Ansiedade e Depressão em Estudantes de Medicina. Revista Brasileira de Educação Médica, 2015; 135-142.

22. WORD HEALTH ORGANIATION (WHO). Menthal health: Strengthening our response. 30 mar. 2018. Disponível em: https://www.who.int/news-room/fact-sheets/detail/mental-health-strengthening-our-response. Acesso em: 23 ago. 21

23. ZHU N, et al. A Novel Coronavirus from Patients with Pneumonia in China. New England Journal of Medicine, 2020; 727-733.

24. ZONTA R, et al. Estratégias de enfrentamento do estresse desenvolvidas por estudantes de Medicina da Universidade Federal de Santa Catarina. Revista Brasileira de Educação Médica, 2006, p.147-153. 